\title{
Successful EUS-guided antegrade stenting for malignant severe biliary obstruction combined with a newly developed plastic stent $\square$
}

\section{다 (1) $(2)$}

\author{
Authors \\ Kazuhiro Minami ${ }^{1}$, Eisuke Iwasaki ${ }^{1}$, Takao Itoi $^{2}$, Seiichiro Fukuhara ${ }^{3}$, Masayasu Horibe ${ }^{1}$, Takashi Seino ${ }^{1}$, Shintaro \\ Kawasaki ${ }^{1}$, Tadashi Katayama ${ }^{1}$, Yoichi Takimoto ${ }^{1}$, Hiroki Tamagawa ${ }^{1}$, Yujiro Machida ${ }^{3}$, Yasuo Hamamoto ${ }^{1}$, Haruhiko \\ Ogata $^{3}$, Takanori Kanai ${ }^{1}$
}

Institutions

1 Division of Gastroenterology and Hepatology, Department of Internal Medicine, Keio University School of Medicine, 35 Shinanomachi, Shinjuku-ku, Tokyo, Japan

2 Department of Gastroenterological Medicine, Tokyo Medical University, 6-7-1, Nishishinjuku, Shinjuku-ku, Tokyo, Japan

3 Center for Diagnostic and Therapeutic Endoscopy, Keio University School of Medicine, 35 Shinanomachi, Shinjuku, Tokyo, Japan

submitted 13.4 .2018

accepted after revision 25.6.2018

\section{Bibliography}

DOI https://doi.org/10.1055/a-0719-4861 |

Endoscopy International Open 2018; 06: E1336-E1339

(c) Georg Thieme Verlag KG Stuttgart · New York

ISSN 2364-3722

Corresponding author

Eisuke Iwasaki, MD, PhD, Division of Gastroenterology and Hepatology, Department of Internal Medicine, Keio
University School of Medicine, 35 Shinanomachi, Shinjuku-ku, Tokyo, Japan

Fax: +81-(0)3-5363-3967

e-iwa@keio.jp

\section{ABSTRACT}

Background and study aims We report the effectiveness of a newly developed plastic stent for preventing bile leakage after endoscopic ultrasonography (EUS)-guided antegrade stenting. This treatment was performed on a 59year-old woman with malignant obstructive jaundice caused by peritoneal metastasis. First, we attempted transpapillary drainage using short-type single-balloon enteroscopy-assisted endoscopic retrograde cholangiopancreatography, but we could not achieve it. We then attempted EUS-guided antegrade stenting through the intrahepatic bile duct from the esophagojejunal anastomosis. We successfully inserted uncovered metallic stents for common bile duct and a newly plastic stent for hepaticojejunostomy from the antegrade approach. There were no adverse events after the treatment.

\section{Introduction}

Acute cholangitis is a fatal disease and doctors need to promptly treat it. However, successful biliary drainage of the gastrointestinal tract after surgery (e. g., Roux-en-Y reconstruction, Billroth II method) is still difficult after enteroscopy. One complicating factor is that the anastomotic region of the bile duct is too far from the mouth to reach with a normal duodenoscope. An alternative method is percutaneous transhepatic drainage. Currently, balloon enteroscopy and endoscopic ultrasoundguided biliary drainage (EUS-BD) are used in some advanced hospitals. Both of these methods have various technical difficulties and complications.
In this case, we performed EUS-guided antegrade stenting (EUS-AG), in which we approached the bile duct from the upper gastrointestinal tract and inserted a biliary stent over Vater's papilla ( $\triangleright$ Video 1$)$. In the case of EUS-AG, one of the potentially severe complications is bile leakage at the puncture site. To prevent this problem, a nasobiliary drainage (NBD) tube is inserted in some hospitals. However, an effective exclusive stent has not been developed for successful biliary drainage. We have hoped for development of such a stent.

A new plastic stent was developed for EUS-guided hepaticogastrostomy. It has a total length of $20 \mathrm{~cm}$, an effective length of $15 \mathrm{~cm}$, and four flanges (two at the distal end and two at the proximal end). The proximal end has a pigtail structure and the distal end is tapered. 


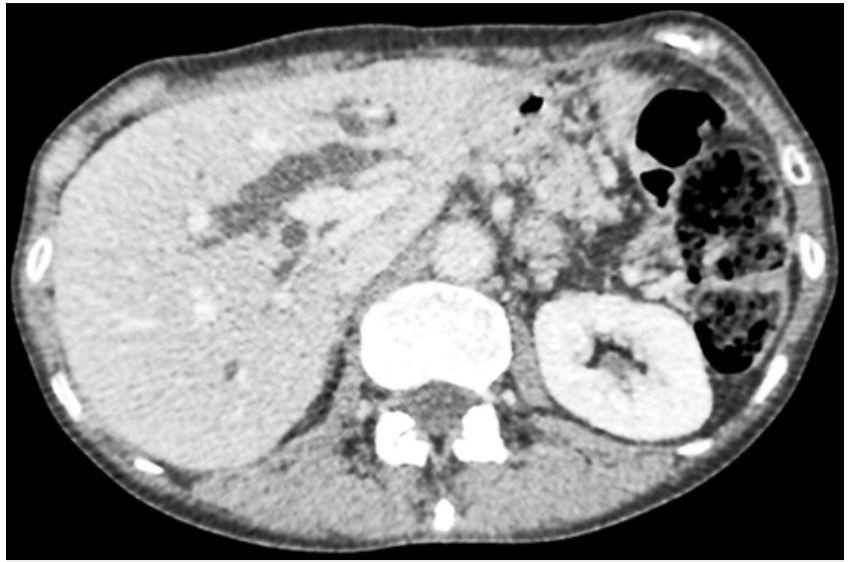

- Fig. 1 Abdominal computed tomography shows dilated intrahepatic bile ducts.

\section{Case report}

A 59-year-old woman received chemotherapy for peritoneal recurrence after total gastrectomy (Roux-en-Y reconstruction) for gastric cancer. During the course of treatment, she was admitted to the hospital with jaundice. An abdominal computed tomography scan showed dilatation of the intrahepatic bile ducts and constriction of the extrahepatic bile duct due to a periportal tumor ( $\triangleright$ Fig. 1). According to these examinations, we diagnosed malignant obstructive jaundice with peritoneal metastasis. Although her physical status was becoming worse, she had no symptom of cholangitis.

We attempted trans-papillary drainage using short-type single-balloon enteroscopy-assisted endoscopic retrograde cholangiopancreatography (ERCP). Selective deep biliary cannulation could not be achieved because of the long segment of severe biliary obstruction and duodenal deformity from peritoneal dissemination. We then attempted EUS-BD for the intrahepatic bile duct from the esophagojejunal anastomosis (convex type, 3.7-mm working channel, GF-UCT260; Olympus Medical Systems, Tokyo, Japan). We punctured the B2 duct with a 19-G needle under fluoroscopic guidance ( $\bullet$ Fig. 2a). In EUS-guided hepaticogastrostomy, puncturing the B3 duct is recommended rather than puncturing B2. This is because B2 puncture may cause transesophageal puncture, which has a risk of pneumomediastinum, pneumothorax, and hemothorax. However, in the case of EUS-AG, the B2 duct is preferable because antegrade procedures are easier to follow [1]. Moreover, we recognized that puncturing the B3 duct was difficult with an EUS image.

Accordingly, we carefully punctured the B2 duct, avoiding the esophagus. We then inserted a standard hydrophilic guidewire (0.025-inch VisiGlide2, Olympus, Tokyo, Japan) into the common bile duct and removed the needle. Thereafter, we inserted a tapered ERCP catheter (MTW Endoskopie, Düsseldorf, Germany) over the wire and contrasted biliary ducts ( $\triangleright$ Fig. $2 \mathbf{b}$ ). At this stage, we considered treatment, EUS-AG, or EUS-rendezvous. Because reaching the papilla took time, and it was expected that the guidewire fell out from the bile duct when we changed the scope to balloon enteroscopy, we chose EUS-AG. We inserted the catheter into the duodenum through the severe stenosis and papilla by using a hydrophilic guidewire. Dilation of the needle tract was performed by using a cautery dilator (6Fr Cysto-Gastro-Set; Endo-Flex GmbH, Voerde, Germany). When selecting stents, we considered that uncovered stents with a slight sheath were better for our case because the stricture was long and tight. We successfully inserted two uncovered metallic stents (Zilver; $8-\mathrm{cm}$ and 6-cm, 10-mm diameter; Cook Japan Co. Tokyo, Japan) from the antegrade approach with 4-mm-diameter balloon predilatation (Hurricane; Boston Scientific, Tokyo, Japan) ( $\triangleright$ Fig. 2c). We further inserted a newly developed temporary $8 \mathrm{Fr}$ single-pigtail plastic stent (IT stent; Gadelius Medical, Tokyo, Japan) from the left intrahepatic bile duct to the esophagus to prevent biliary leakage ( $\triangleright$ Fig. $2 d$ ).

The day after the procedure, the patient resumed drinking water. The patient's jaundice and symptoms markedly improved without any adverse events, such as migration of the plastic stent or biliary leakage. When she left the hospital, she could eat solid food. Normally, this stent can be removed after approximately 2 weeks. This period is required for fistulation between the bile duct and gastrointestinal tract. However, in our case, the patient's condition was terminal and she had no subjective symptoms. Therefore, we did not remove the plastic stent. After 3 months, she died without any biliary tract problems.

\section{Discussion}

The first report on EUS-BD was by Giovannini et al. in 2001 [2]. Various EUS-BD techniques have been developed since then. Placement of a self-expanding metal stent for malignant biliary obstruction with EUS-AG was first reported by Fujita et al. in 2007 [3]. One-step EUS-AG was also reported by Binmoeller et al. in 2010 [4]. Kawakubo et al summarized the clinical results of EUS-AG in 2015 [5]. They reported that the success rate was $87 \%(67 / 77)$ and the complication rate was $13 \%$ (10/77).

Although EUS-AG has a high technical success rate, some complications have been reported, such as bleeding, bile leakage (peritonitis), stent migration, mediastinitis, and biloma. Although there are few studies on EUS-AG, it is thought to be best for preventing bile leakage by placing a NBD tube after EUSguided antegrade balloon dilatation [6]. We believe that NBD is also effective after EUS-guided antegrade biliary stenting, and the access route can be maintained when reintervention is required. However, an NBD tube leads to discomfort for patients and decreases their activity. The partially/fully covered self-expanding metal stent is also considered for covering hepaticojejunostomy route, however, there is a risk of mucosal injury. We consider a plastic stent better. The efficacy of this newly developed plastic stent for EUS-guided hepaticogastrostomy stenting was reported in 2015 [7]. In our case, we demonstrated the efficacy of EUS-AG with temporary hepatico-esophageal stenting for preventing bile leakage. 

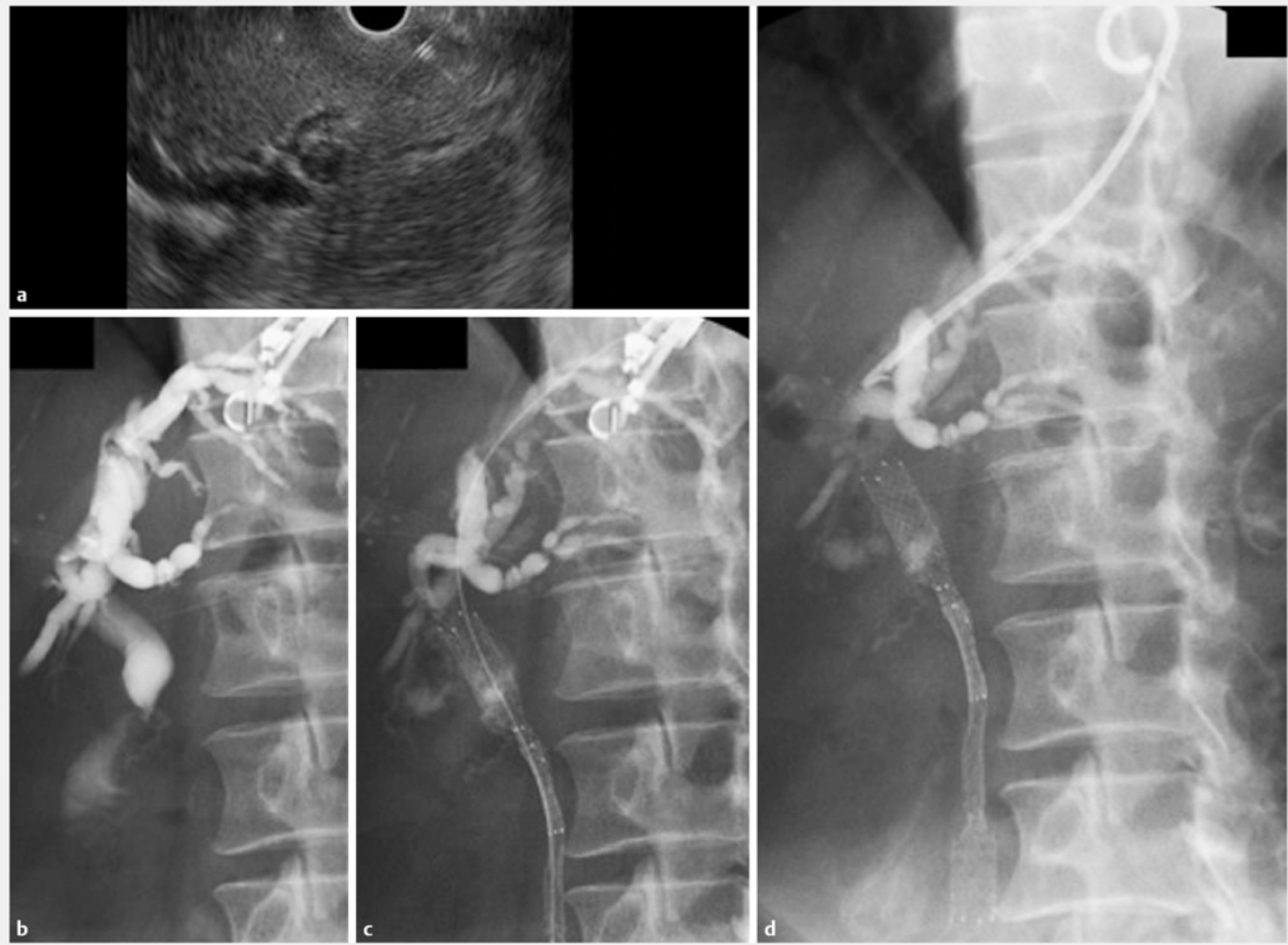

b
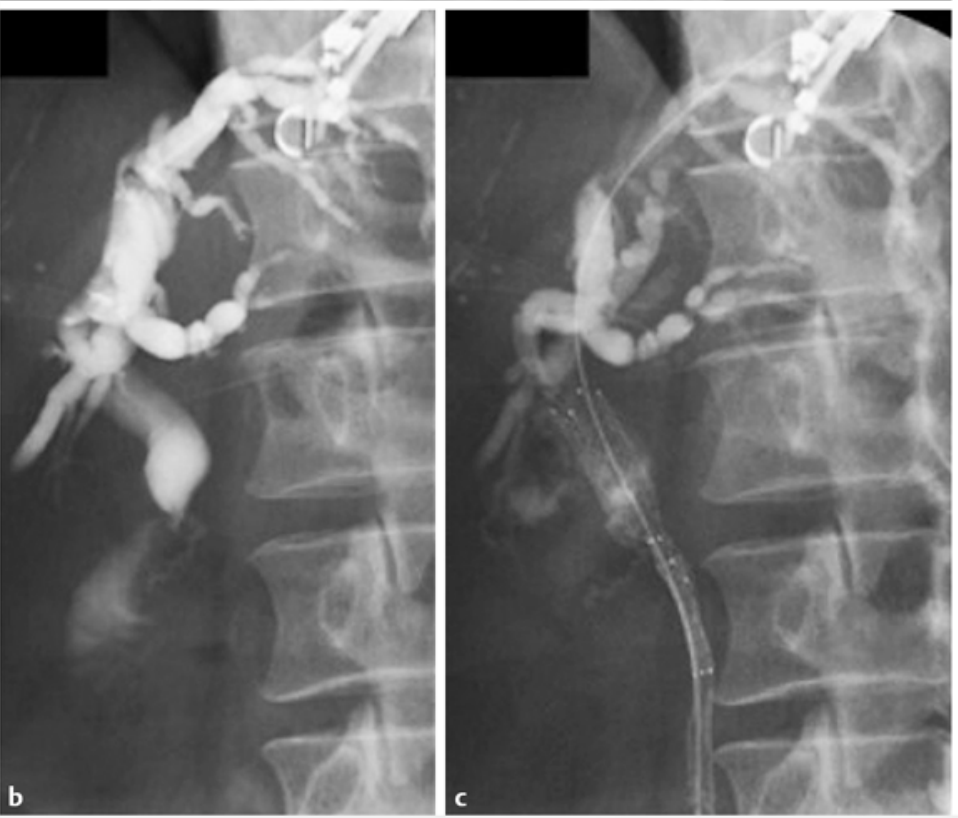

- Fig. 2 a Punctured B2 duct with a 19-G needle. $\mathbf{b}$ Insertion of the catheter over the wire and contrasted biliary ducts. c Successfully inserted uncovered metallic stents using the antegrade technique with balloon predilatation. $\mathbf{d}$ Insertion of a temporary single pigtail plastic stent from the left intrahepatic bile duct to the esophagus to prevent biliary leakage.

\section{Conclusion}

We consider that this newly developed stent can reduce complications related to EUS-AG. The usual tube stent tends to come out easily, but this plastic stent remains in place because of its shape and anti-migration flaps. Moreover, this plastic stent has higher cost performance and facilitates easier reintervention than metallic stents, and it is beneficial for patients from a physiological perspective.

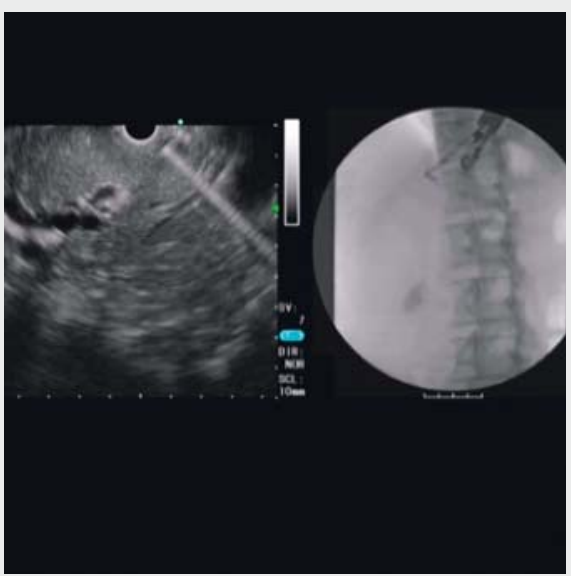

$\nabla$ Video 1 EUS-AG with newly developed stent. 


\section{Competing interests}

None

\section{References}

[1] Itoi T, Sofuni A, Tsuchiya T et al. Endoscopic ultrasonography-guided transhepatic antegrade stone removal in patients with surgically altered anatomy:case series and technical review(with videos). J Hepatobiliary Pancreat Sci 2014; 21: 86 - 93

[2] Giovannini M, Moutardier V, Pesenti C et al. Endoscopic ultrasoundguided bilioduodenal anastomosis: a new technique for biliary drainage. Endoscopy 2001; 33: $898-900$
[3] Fujita N, Noda Y, Kobayashi G et al. Histological changes at an endosonography-guided biliary drainage site: a case report. World J Gastroenterol 2007; 13: $5512-5515$

[4] Nguyen-Tang T, Binmoeller KF, Sanchez-Yague A et al. Endoscopic ultrasound (EUS)-guided transhepatic anterograde self-expandable metal stent (SEMS) placement across malignant biliary obstruction. Endoscopy 2010; 42: $232-236$

[5] Kawakubo K, Kawakami H, Kuwatani M et al. Recent advances in endoscopic ultrasonography-guided biliary interventions. World J Gastroenterol 2015; 21: $9494-9502$

[6] Iwashita T, Yasuda I, Doi S et al. Endoscopic ultrasound-guided antegrade treatments for biliary disorders in patients with surgically altered anatomy. Dig Dis Sci 2013; 58: 2417 - 2422

[7] Umeda J, Itoi T, Tsuchiya T et al. A newly designed plastic stent for EUS-guided hepaticogastrostomy: a prospective preliminary feasibility study (with videos). Gastrointest Endosc 2015; 82: 390 - 396 